\title{
Study on Differences between Chinese and Foreign Vocational Trainings of Aged Care Workers
}

\author{
Dapeng Cong \\ Yantai Nanshan University \\ Yantai, Shandong, China 265713
}

\author{
Shengqun Wang \\ Yantai Nanshan University \\ Yantai, Shandong, China 265713
}

\author{
Yan Gao \\ Yantai Nanshan University \\ Yantai, Shandong, China 265713
}

\begin{abstract}
Entering the 21st century, worldwide aging tendency becomes increasingly serious and prominent. The needs of the old for old-age care services increase sharply and the demands for higher quality services has been instantly increased. America, as a forerunner of stepping into aging society, has formed a relatively completed old-age care services system during the long-term exploration. By comparing the gap of current situation of old-age care profession, occupational description and occupational reputation between two countries, some referable suggestions on improvement of the life quality of old people, promotion of the healthy development of old-age care, and facilitation of the social harmony, can be provided.
\end{abstract}

Keywords-old-age care; vocational education; current development situation; China; abroad; contrast

\section{INTRODUCTION}

It can be learned from the statistical data of the United Nations population development foundation, by 2012, about 810 million people around the world has reached or exceeded 60 years old, which occupies $11 \%$ of the world's population, that is to say, there is one who are aged 60 or more than 60 in every nine people; It is predicted that by 2050 , the aging population will reach 2.03 billion accounting for more than $22 \%$ of the total population, which means that there is one who reaches or over 60 in every five people. With the degree of global ageing increasing and deepening, the resulted old-age nursing problems are increasingly prominent. Attaching importance to the development of nursing services has become a worldwide concern in order to effectively cope with surging pension service demands. After a long period of exploration practice, some foreign developed countries have accumulated a large number of practical experiences in the development of old-age nursing career.

The aging society in our country is relatively late, and oldage nursing system is still in its initial stage of construction and the old-age nursing profession also needs to be further developed and completed. By comparing the old-age nursing career development at home and abroad on the current situation, the author expects to borrow the experience of
America for promoting the development of China old-age nursing career.

\section{PROFESSIONALIZATION OF FOREIGN OLD-CARE NURSES}

\section{A. Japan Old-care Training System}

Many foreign countries started the development of old-care career earlier than China, therefore, which creates actual and practical referable cases for the development of Chinese oldcare nurses in the future. For example, in Japan social welfare worker has become a special job. Qualification admittance system has been implemented aiming at all various personnel in welfare institution in Japan since 1971. The system requires the manager of social welfare institution must: Take relevant elective courses and graduate from universities and colleges; attend the specific test for the staff of social welfare institution. In 1997, Japanese Ministry of Education and Ministry of Health and Welfare officially brought community care into the teaching programme and opened community care colleges and ensured the cultivation and recognition of career qualification system of community nurses by law.

Since 2000, Japanese government has implemented nursing insurance system operated by government to be confronted with the coming aging society. At the early stage of implementation, about 48,000 "special care support members" are needed in the nationwide. Besides that, although "welfare care nurses" for assisting the life of the old and disabled, and "Middle-aged and old life consultant" for accepting and disposing the life problems consulting of middle-aged and old people appear as a new qualification, they are also popular in Japanese society. This phenomenon enables to grow to the service career which can absorb a large number of employees. Thus, Japanese society can provide more job opportunities and also a sense of safety for people owning relevant qualifications. Japanese nursing staff has a good public reputation with a constantly rising social status. In recent years, passing the qualification exam of nursing staff has been regarded as a symbol to display their ability, status and kindness by Japanese women. 
Two distinct features occurred in the development process of Japan old-care career: First, the cultivation of Japanese nursing staff has been linked with schools, which ensured the specialty of the caregivers from the source and assess system. However, because the lateness of nurse career development in China, it is unrealistic to reach that goal in a short term. It is a long-term process to open corresponding courses in universities and colleges for ensuring the source of professional old-care nursing assistants. Second, Japanese oldcare nursing staff owns a high social status which is dependent on their high professional quality. Various of professional certification systems have built a high threshold for the profession of old-care nursing assistants. Nursing assistant has become a respectable profession as lawyers with high and strict demands and excellent competitive abilities. In China, the relevant professional recognition system just starts up. Most people of our society still consider the nursing assistants as babysitters and housekeeping personnel, etc. Deserved attention has not been paid to the treatment problems of nursing staff. Low salaries and high payment of this profession has made it equal to the general "manual labor", thus, naturally it has no much social recognition, which directly impacts the improvement of professional level of old-care nursing staff[2].

Except Japan, America also owns an earlier development in this career: In 1966, America opened old-care major courses in Duke University. Nurses graduating from this major are able, after passing the qualification examination held by American Nurses Association, to become a practitioner of old-care major. The nursing staff grading system of the United States is quite completed. In the long-term old-care survey of the countries, nursing staff is divided into three types ; Primary nursing staff taking the full responsibilities of caring for patients; Primary nursing staff receiving informal (no consideration) or formal (with remuneration) assistances; Minor nursing staff not responsible for patents. Nursing staff and visited servicers spend 18 hours for each week providing nursing services. Among them, about one fifth provides sustaining nursing or at least 40-hour caring per week. Although the old-care career of the United States has been developed, the old-care nursing staff is still in shortage due to the pressure of old caring [3]. Therefore, America has set the qualification examination of registered nurse in 29 countries and regions, so that the managers of nursing institution for the aged can take his position only after learning the relevant professional knowledge and obtaining the certifications.

\section{B. Old-age Care Training System of the United States}

The development status of American old-age care nursing staff has following features: First, like Japan, since the related majors have been opened in colleges and universities, nursing assistants can become professional personnel after passing the relevant examination. On the contrary, at present China still remains the system that people intending to work on this career are trained and assessed first and then can take the position as old-aged care nursing staff after passing the assessment. Second, the nursing personnel grade system of the United States is quite completed. And China will develop nursing career orienting to the direction; however, it is difficult to achieve the goal within a short term considering the reality [4].
The classification of nursing personnel is impossible for now because qualifying standard of Chinese old-aged care nursing personnel was just issued in 2002. It's only the matter of time. The related departments have realized the importance of professionally grading nursing personnel and set four professional grades in State Professional Standard of Nursing Personnel, which are, primary (national vocational qualification level five), intermediate ( national vocational qualification level four), advanced (national vocational qualification level three) and technician (National vocational qualification level two). Third, American nursing personnel has an averaged working time, which differs from the current situation of Nanjing City where adopts the principle of "able person should do more work". That is to say, some nursing personnel having more abilities and higher professional skills can care for more old people. Otherwise, the new staff can care for one or two old people and spend his rest time in skill training for caring for more old people in the future. Fourth, American calculates its professional nursing personnel in worldwide and sets qualification examination institutions in other regions. This is a peculiar method of America. Since China and other countries cannot borrow the method since they do not have that economic strength and international influence like America. However, besides that, China has encountered the same problems with America [5]. For example, some writings studying on gender issues mention that: In America, most of nursing personnel is women which occupies about three fourths of the whole primary nursing staff. Comparatively speaking, Chinese nursing personnel also faces the same "gender embarrassment" problem, and the shortage of male nursing personnel has a strong impacts on the caring quality of male old people.

\section{DOMESTIC OLD-AGED CARE TRAINING SYSTEM OF NURSING PERSONNEL}

In order to response for the social issues brought by the pressure of social provision for the added, old-aged care nursing assistants become social urgently required talents day by day. Many places in our country released related measures and some of them have achieved valid outcome: The first session of nursing personnel training class, opened by old people's home of Tianjin City, completed its courses in Mar. 1st, 2004. 65 students obtained Vocational Qualification of Old-age Home-based Nursing and Physical Health Certification. More than 200,000 old people in Tianjin City have the demands for nursing, while the city faces a serious shortage of trained nursing personnel. Old people's homes utilize nursing institutions for the aged to conduct professional training on nursing personnel. Allowance of the nursing personnel entering families is the effective way to improve service quality. The training classes separate the family nursing of old people from housekeeping work by cooperation with entrepreneurship training center of Tianjin municipal Labor and Social Security Bureau. They provide free training for laid-off workers on the knowledge of health care, and implement professional training, industry supervision and acceptance mechanism. Fushun City, Liaoning Province has passed the training and trail operation stage aiming at old-aged home-based nursing personnel and officially launched "home care" work. Government should purchase services for old 
people, and arranges the 99 nursing personnel of first batch to hold the public welfare position to provide old people the visiting services of housekeeping, buying and supplying meals, and accompanying. Qingdao City opened jointly the intermediate training class of old-age nursing home with Singapore Tsao Foundation and built a professional old-age nursing team in order to fit the development of aging social. The basic way of training is divided into four procedures: The first step is to recruit talents from the society. People who have love heart and patience and with an age from 30 to 55 years old can apply for it; the second step is to conduct professional training of the selected talents for about three months. The training content mainly includes ideology and morality, basic nursing technique, caring and nursing practice training and so on. By a serial of professional training, improve the nursing capacity and service level of nursing personnel; the third step is to strictly conduct assessment. After passing the examination and training internship, training certificate and health certification can be released personnel and then they can hold the position; the fourth step is that, personnel who passing the training and assessment are able to enjoy the staff's treatment of the unit. The service remuneration of nursing personnel is 6.6 yuan for per hour; if nursing personnel works in the headquarter of nursing home, its service remuneration should be determined according to nurse the intensity and difficulty of nursing of the practical work, to ensure the stability of old-age nursing team and the persistent development of home-based services [7].

The advanced methods of government of every area are to formulate related measures first and purchase services, and then open old-age nursing training class and so on. Aiming at the professionalization problem of nursing personnel, Nanjing City also took some corresponding measures and launched the old-age nursing training project in 2005 , on the purpose of making over 1,000 nursing personnel of 190 nursing institutions for the aged employ with certifications. With the fastening of social aging, great changes of old people's caring and nursing awareness have taken place, and more and more old people require spending their twilight years in nursing homes.

The degree of specialism of Chinese aged care works is not high yet. Home aged care staff's degree of specialism is higher than that of institutional aged care staff. The writer believes that the reason is that: Firstly, nursing object of home care and institutional care: differences of the olds. Most of the old people of institutional care are without self-care ability and in a weak position. While the demands of old people of home care to the care staff is limited. Secondly, the status of aged care staff is different. Home aged care staff can express their opinions freely during their daily work and sum up the experience of the work in time. So their enthusiasm and initiative of work is bigger. Thirdly, the origin of aged care worker is different, which leads to a different starting point for the development of home aged care worker and institutional aged care worker. The difference of care staff on cultural quality, ideological quality and acceptance will lead to different professional training in the early stage. Fourthly, work environment and interpersonal relationship of aged care staff are different. Leaders and nursing staffs of home care are in a harmonious and equal relationship. They learn together and are equal in status during the process of training. While in institutional care, the care staff is obviously in a weak position and must obey the lead and command of the person in charge.

\section{REFERENCES}

[1] Qin Li, Wang Mingzhu. Research on Assessment Indicator System of Old-care Services Policy-Take Yanbian Autonomous Prefecture as Example [J]. Heilongjiang Annals. 2014 (11).

[2] Yu Zishu, Zhao Danhong. Enlightenment on Dealing with Social Aging of Chinese Society by Carry forward the Traditional Blessing Culture [J]. Industry and Technology Forum. 2014 (20).

[3] Zhao Xiaodong, Bao Qingyu. Cultural Old-aged Caring and Old-aged Caring Culture-Analysis on the Connotative Meaning of Cultural Oldaged Caring [J]. Popular Literature. 2014 (21).

[4] Yang Ruiqi, Ma Baoxiang. A Brief Introduction of the Educational and Inherited Function of Cultural Activity of the Old-Take Cultural Activity of the Old for Example [J]. Popular Literature. 2014 (15).

[5] Hou Jingang. The Republic of China under the View of Social Gender-Research Centered by Beneficial Friend Pictorial Magazine [J]. Popular Literature. 2014 (20).

[6] Huang Chuncheng. Rational Usage of "Old and Young" Staff [J]. Modern Enterprise Culture (Early). 2014(10).

[7] HeJingmei, Wang Haiyan, Ma Yunjun. Feasibility Research on Property Management Company Participating in Home-based Caring for the Aged [J]. Modern Commercial Trade Industry. 2014 (24) (2014 (05).

[8] A Zhifang, A zhiqin. Discussion on the Spiritual Consolation Problems of Colleges and Universities Retired Teachers [J]. HeBei North University Journal (Social sciences). 2014 (05).

[9] Qiu Ming. Design and Implementation of Community Service for Home Care of Eldly [J]. Xiangyang Vocational and Technical College Journal. 2014 (06).

[10] Wang Weizhi. Practice and Thought on Gansu Old-age Service System Construction [J]. China's Finance. 2014 (19). 